\title{
一类高次缺插值样条函数
}

\author{
陈天平 \\ (复 旦大学)
}

在文献 [1]中, Meir 和 Sharma 提出了一种五次缺插值样条函数, 文献 [2,3] 改进了文献 [1] 中的结果.

最近,沙震用类似方法讨论了七次、十一次样条. 本文目的是给出一种一般的处理方法, 不仅大大改进了已有结果, 而且可用来讨论一般的插值偶次导数的样条.

在 1 中, 通过 Hermite 插值样条讨论一种 $\mathrm{H}-\mathrm{B}$ 插值样条给出收玫速度估计和渐近展 开.

在 $\$ 2$ 中, 用 $\$ 1$ 中结果讨论一类高次缺插值样条, 给出收玫速度估计、渐近展开及饱和函 数类.

$\S 1$ 设 $f(x) \in C^{r}[0,1], x_{\nu}=\frac{\nu}{n}, v=0,1, \cdots, n$ 是区间 $[0,1]$ 的一个分划, $h=\frac{1}{n}$, $S_{n, 1}(x)=S_{n, 1}(f, x)$ 为满足下述条件的 Hermite 插值样条函数:

(i) 在 $\left[x_{\nu}, x_{v+1}\right]$ 上, $\nu=0,1, \cdots, n-1, S_{n, 1}(x)$ 是 $2 r+1$ 次多项式;

(ii) $S_{n, 1}^{(p)}\left(x_{v}\right)=f^{(p)}\left(x_{v}\right), p=0,1, \cdots, r$.

在文献[4]中, 我们证明了

定理 $\mathbf{A}$ 设 $f(x) \in C^{s}[0,1](r \leqslant s \leqslant 2 r+1)$, 则当 $q \leqslant s$ 时,

$$
\left\|S_{n .1}^{(q)}(f ; x)-f^{(q)}(x)\right\|=O\left(h^{s-q} \omega\left(f^{(s)} ; h\right)\right) \text {. }
$$

如 $f(x) \in C^{2 r+2}[0,1]$, 则

$$
f^{(q)}(x)-S_{n, 1}^{(q)}(f ; x)=h^{2 r+2-q} f^{(2 r+2)}\left(x_{v}\right) R^{(q)}\left(\frac{x-x_{\nu}}{h}\right)+O\left(h^{2 r+2-q} \omega\left(f^{(2 r+2)} ; h\right)\right),
$$

其中 $R(x)=x^{r+1}(x-1)^{r+1} /(2 r+2)$ !

设 $S_{n, 2}(x)=S_{n, 2}(f ; x)$ 为满足下述条件的 $\mathrm{H}-\mathrm{B}$ 插值样条,

(i) 在 $\left[x_{v}, x_{\nu+1}\right]$ 上, $\nu=0,1, \cdots, n-1, S_{n, 2}(x)$ 是 $2 r+1$ 次多项式;

(ii) $S_{n, 2}^{(2 p)}\left(x_{v}\right)=f^{(2 p)}\left(x_{v}\right), \nu=0,1, \cdots, n, p=0,1, \cdots, r$.

当 $x \in\left(x_{v}, x_{v+1}\right)$ 时,

$$
S_{n, 2}(f ; x)=\sum_{i=0}^{1} \sum_{j=0}^{r} h^{2 j} f^{(2 j)}\left(x_{v+i}\right) v_{i, j}\left(\frac{x-x_{v}}{h}\right),
$$

其中 $v_{i, j}(x)(i=0,1, j=0,1, \cdots, r)$ 为满足下列条件的 $2 r+1$ 次多项式

$$
v_{i, j}^{\left(2^{l}\right)}(k)=\delta_{i, k} \cdot \delta_{j, l} \quad(i, k=0,1, j, l=0,1, \cdots, r) .
$$

直接验证易得

本文 1980 年 8 月 28 日收到. 


$$
\left\{\begin{array}{l}
1 \equiv v_{0,0}(x)+v_{1,0}(x), \\
x \equiv v_{1,0}(x), \\
\frac{x^{k}}{k !} \equiv\left\{\begin{array}{l}
v_{0, \frac{k}{2}}(x)+\sum_{p=0}^{\frac{k}{2}} \frac{v_{1, p}(x)}{(k-2 p) !} \text { 当 } k \text { 是偶数, } \\
{\left[\frac{k}{2}\right\rceil \frac{v_{1, p}(x)}{(k-2 p) !}}
\end{array} \text { 当 } k\right. \text { 是奇数. }
\end{array}\right.
$$

类似定理 $\mathrm{A}$, 我们可以证明

定理 1 设 $f(x) \in C^{s}[0,1](s=2 r, 2 r+1)$, 则当 $q \leqslant s$ 时,

$$
\left\|S_{n, 2}^{(q)}(f ; x)-f^{(q)}(x)\right\|=O\left(h^{s-q} \omega\left(f^{(s)} ; h\right)\right),
$$

当 $S=2 r+2$ 时,成立着

$$
\begin{gathered}
f^{(q)}(x)-S_{n, 2}^{(q)}(x)=h^{2 r+2-q} Q^{(q)}\left(\frac{x-x_{\nu}}{h}\right) f^{(2 r+2)}(x)+O\left(h^{2 r+2-q} \omega\left(f^{(2 r+2)} ; h\right)\right) \\
\left(x_{\nu}<x<x_{\nu+1}\right),
\end{gathered}
$$

其中

$$
Q(x)=R(x)-\sum_{p=0}^{r}\left[R^{(2 p)}(0) v_{0, p}(x)+R^{(2 p)}(1) v_{1, p}(x)\right] .
$$

证 设 $S_{n, 1}(f ; x)$ 为 Hermite 插值样条, 则

$$
\begin{aligned}
S_{n, 2}(f ; x)= & S_{n, 1}(f ; x)+\sum_{p=0}^{r} h^{2 p}\left\{\left[f^{(2 p)}\left(x_{\nu}\right)-S_{n, 1}^{(2 p)}\left(x_{\nu}+\right)\right] v_{0, p}\left(\frac{x-x_{\nu}}{h}\right)\right. \\
& \left.+\left[f^{(2 p)}\left(x_{\nu+1}\right)-S_{n, 1}^{(2 p)}\left(x_{\nu+1}-\right)\right] v_{1, p}\left(\frac{x-x_{\nu}}{h}\right)\right\} .
\end{aligned}
$$

由定理 A, 当 $s=2 r, 2 r+1$ 时,

$$
\begin{aligned}
\left\|S_{n, 2}^{(q)}(f ; x)-f^{(q)}(x)\right\| & =\left\|S_{n, 1}^{(q)}(f ; x)-f^{(q)}(x)\right\|+O\left(h^{s-q} \omega\left(f^{(s)} ; h\right)\right) \\
& =O\left(h^{s-q} \omega\left(f^{(s)} ; h\right)\right),
\end{aligned}
$$

当 $s=2 r+2$ 时, 把(2)式代人(6)式即得(5)式. 证毕.

$\$ 2$ 设 $x_{\nu}=\frac{v}{n}, r=3 k, S_{n}(x)$ 为以 $x_{\nu}$ 为节点的 $2 r+1$ 次样条函数, 它满足

(i) $S_{n}(x) \in C^{2 r-2 k}[0,1]$; (ii) 在 $\left[x_{\nu}, x_{\nu+1}\right]$ 上, $\nu=0,1, \cdots, n-1$, 是 $2 r+1$ 次多项 式; (iii) $S_{n}^{(2 p)}\left(x_{v}\right)=f^{(2 p)}\left(x_{v}\right), p=0,1, \cdots, r-k, \nu=0,1, \cdots, n$; (iv) $S_{n}^{(2 i+1)}(0)=f^{(2 i+1)}(0)$, $i=0,1, \cdots, 2 k-1$.

我们证㛣

定理 2 如果基函数 $v_{i, j}(x)$ 满足

$$
\operatorname{det}\left(\begin{array}{lllllll}
v_{0, r-k+1}^{\prime}(1) & v_{1, r-k+1}^{\prime}(1) & v_{0, r-k+2}^{\prime}(1) & v_{1, r-k+2}^{\prime}(1) & \cdots & v_{0, r}^{\prime}(1) & v_{1, r}^{\prime}(1) \\
v_{0, r-k+1}^{\prime 3)}(1) & v_{1, r-k+1}^{(3)}(1) & v_{0, r-k+2}^{(3)}(1) & v_{1, r-k+2}^{(3)}(1) & \cdots & v_{0, r}^{(3)}(1) & v_{1, r}^{(3)}(1) \\
& & & \cdots \cdots & & & \\
& & & \cdots & \cdots & \\
v_{0, r-k+1}^{2(r-k)-1}(1) & v_{1, r-k+1}^{2(r-k)-1}(1) & v_{0, r-k+2}^{2(r)-k)-1}(1) & v_{1, r-k+2}^{2(r-k)-1}(1) & \cdots & v_{0, r}^{2(r-k)-1}(1) & v_{1, r}^{2(r-k)-1}(1)
\end{array}\right) \neq 0,
$$

$f(x) \in C^{s}[0,1](2(r-k) \leqslant s \leqslant 2 r+1)$, 则

$$
\left\|S_{n}^{(q)}(f ; x)-f^{(q)}(x)\right\|=O\left(h^{s-1-q} \omega\left(f^{(s)} ; h\right)\right), \quad(q \leqslant s-1) .
$$


当 $f(x) \in C^{2 r+2}[0,1]$ 时,存在 $2 r+2$ 次多项式 $C(x)$ 满足, 当 $x \in\left[x_{v}, x_{v+1}\right]$ 时,

$$
\begin{aligned}
f^{(q)}(x)= & S_{n}^{(q)}(x)+C^{(q)}\left(\frac{x-x_{v}}{h}\right)\left[f^{(2 r+1)}\left(x_{v}\right)-f^{(2 r+1)}\left(x_{0}\right)\right] h^{2 r+1-q} \\
& +O\left(h^{2 r+1-q} \omega\left(f^{(2 r+2)} ; h\right)\right) .
\end{aligned}
$$

证 由 $v_{i, j}(x)$ 的定义, $v_{0, j}(x)=v_{1, j}(1-x)$, 故当 $q$ 是奇数时,

$$
v_{0, j}^{(q)}(x)=-v_{1, j}^{(q)}(1-x) \text {. }
$$

首先, 设 $s=2 r+1$. 由定义, 当 $x \in\left[x_{\nu}, x_{\nu+1}\right]$ 时,

$$
\begin{aligned}
S_{n}(x)= & \sum_{p=0}^{r-k} h^{2 p}\left[f^{(2 p)}\left(x_{\nu}\right) v_{0, p}\left(\frac{x-x_{\nu}}{h}\right)+f^{(2 p)}\left(x_{\nu+1}\right) v_{1, p}\left(\frac{x-x_{\nu}}{h}\right)\right] \\
& +\sum_{p=r-k+1}^{r} h^{2 p}\left[S_{n}^{(2 p)}\left(x_{\nu}+\right) v_{0, p}\left(\frac{x-x_{\nu}}{h}\right)+S_{n}^{(2 p)}\left(x_{\nu+1}-\right) v_{1, p}\left(\frac{x-x_{\nu}}{h}\right)\right] .
\end{aligned}
$$

由是得到连续性方程

$$
\begin{gathered}
\sum_{p=r-k+1}^{r} h^{2 p}\left\{\left[S_{n}^{(2 p)}\left(x_{\nu}+\right)-f^{(2 p)}\left(x_{v}\right)+S_{n}^{(2 p)}\left(x_{v+2}-\right)-f^{(2 p)}\left(x_{v+2}\right)\right] v_{0, p}^{(2 i+1)}(1)\right. \\
\left.+\left[S_{n}^{(2 p)}\left(x_{v+1}-\right)-f^{(2 p)}\left(x_{v+1}\right)+S_{n}^{(2 p)}\left(x_{v+1}+\right)-f^{(2 p)}\left(x_{\nu+1}\right)\right] v_{,, p}^{(2 i+1)}(1)\right\} \\
=h^{2 i+1}\left[S_{n, 2}^{(2 i+1)}\left(x_{v+1}+\right)-S_{n, 2}^{(2 i+1)}\left(x_{\nu+1}-\right)\right] . \\
\left(\begin{array}{l}
i=0,1, \ldots, r-k-1 \\
v=0,1, \cdots, n-2
\end{array}\right)
\end{gathered}
$$

固定 $\boldsymbol{\nu}$, 相应的系数矩阵是 $(r-k) \times(r-k)$ 阶矩阵 $(r=3 k)$. 由定理的假设它是非奇 导的. 而由定理 $1,(8)$ 式右端等于 $O\left(h^{2 r+1} \omega\left(f^{(2 r+1)} ; h\right)\right)$, 故可解出

$$
\left\{\begin{array}{c}
{\left[S_{n}^{(2 p)}\left(x_{\nu}+\right)-f^{(2 p)}\left(x_{\nu}\right)\right]+\left[S_{n}^{(2 p)}\left(x_{\nu+2}-\right)-f^{(2 p)}\left(x_{v+2}\right)\right]=O\left(h^{2 r+1-2 p} \omega\left(f^{(2 r+1)} ; h\right)\right),} \\
{\left[S_{n}^{(2 p)}\left(x_{\nu+1}-\right)-f^{(2 p)}\left(x_{\nu+1}\right)\right]+\left[S_{n}^{(2 p)}\left(x_{\nu+1}+\right)-f^{(2 p)}\left(x_{\nu+1}\right)\right]=O\left(h^{2 r+1-2 p} \omega\left(f^{(2 r+1)} ; h\right)\right) .} \\
(p=r-k+1, \cdots, r, \nu=0,1, \cdots, n-2)
\end{array}\right.
$$

由端点条件

$$
\begin{aligned}
& \sum_{p=r-k+1}^{r} h^{2 p}\left\{\left[S_{n}^{(2 p)}\left(x_{0}+\right)-f^{(2 p)}\left(x_{0}\right)\right] v_{0, p}^{(2 i+1)}(0)+\left[S_{n}^{(2 p)}\left(x_{1}-\right)-f^{(2 p)}\left(x_{1}\right)\right] v_{1, p}^{(2 i+1)}(0)\right\} \\
= & h^{2 i+1} f^{(2 i+1)}(0)-\sum_{p=0}^{r} h^{2 p}\left[f^{(2 p)}\left(x_{0}\right) v_{c, p}^{(2 i+1)}(0)+f^{(2 p)}\left(x_{1}\right) v_{1, p}^{(2 i+1)}(0)\right] \\
= & O\left(h^{(2 r+1)} \omega\left(f^{(2 r+1)} ; h\right)\right) \quad(i=0,1, \cdots, r-k-1) .
\end{aligned}
$$

由是

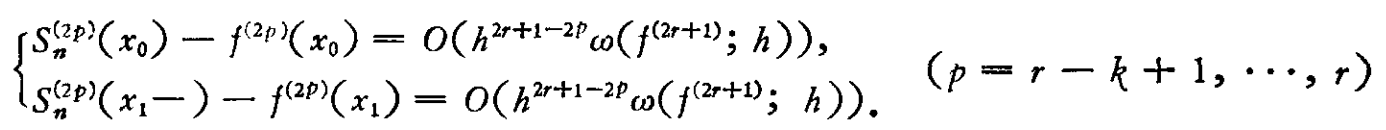

由(9)式和(10)式即得对一切 $\nu=0,1, \cdots, n$,

$$
\begin{gathered}
S_{n}^{(2 p)}\left(x_{\nu} \pm\right)-f^{(2 p)}\left(x_{\nu}\right)=O\left(h^{2 r-2 p} \omega\left(f^{(2 r+1)} ; h\right)\right), \\
(p=r-k+1, \cdots, r)
\end{gathered}
$$

利用定理 1 , 立即可知, $s=2 r+1$ 时定理成立.

次设 $f(x) \in C^{2 r+2}[0,1]$, 把定理 1 中(5)式代入(8)式

$$
\sum_{p=r-k+1}^{r} h^{2 p}\left\{\left[S_{n}^{(2 p)}\left(x_{\nu}+\right)-f^{(2 p)}\left(x_{\nu}\right)+S_{n}^{(2 p)}\left(x_{\nu+2}-\right)-f^{(2 p)}\left(x_{\nu+2}\right)\right] v_{0, q}^{(2 i+1)}(1)\right.
$$




$$
\begin{gathered}
\left.+\left[S_{n}^{(p p)}\left(x_{\nu+1}-\right)-f^{(2 p)}\left(x_{\nu+1}\right)+S_{n}^{(2 p)}\left(x_{\nu+1}+\right)-f^{(2 p)}\left(x_{\nu+1}\right)\right] v_{1, p}^{(2 i+1)}(1)\right\} \\
=h^{2 r+2} f^{(2 r+2)}\left(x_{\nu+1}\right)\left[Q^{(2 i+1)}(1)-Q^{(2 i+1)}(0)\right]+O\left(h^{2 r+2} \omega\left(f^{(2 r+2)} ; h\right)\right) . \\
\left(\begin{array}{l}
i=0,1, \cdots, r-k-1 \\
v=0,1, \cdots, n-2
\end{array}\right)
\end{gathered}
$$

结合端点条件, $S_{n}(f ; x)$ 是唯一决定的. 适当减去一个 $2 r+1$ 次多项式可设 $f^{(2 r+2)}(0)=0$, 故 $f^{(2 r+2)}(x)=O\left(h^{-1} \omega\left(f^{(2 r+2)} ; h\right)\right)$. 因此, 由 (11) 式, 存在常数 $C_{p, 1}, C_{p, 2}$, 使

$$
\begin{aligned}
S_{n}^{(2 p)}\left(x_{\nu}+\right) & -f^{(2 p)}\left(x_{\nu}\right)=C_{p, 1} h^{2 r+1-2 p}\left[f^{(2 r+1)}\left(x_{\nu}\right)-f^{(2 r+1)}\left(x_{0}\right)\right] \\
& +O\left(h^{2 r+1-2 p} \omega\left(f^{(2 r+2)} ; h\right)\right), \\
S_{n}^{(2 p)}\left(x_{\nu}-\right) & -f^{(2 p)}\left(x_{\nu}\right)=C_{p, 2} h^{2 r+1-2 p}\left[f^{(2 r+1)}\left(x_{\nu}\right)-f^{(2 r+1)}\left(x_{0}\right)\right] \\
& +O\left(h^{2 r+1-2 p} \omega\left(f^{(2 r+2)} ; h\right)\right) .
\end{aligned}
$$

代人定理 1 知, 存在着 $2 r+2$ 次多项式 $C(x)$, 使当 $x \in\left(x_{v}, x_{v+1}\right)$,

$$
\begin{aligned}
f^{(q)}(x)= & S_{n}^{(q)}(x)-C^{(q)}\left(\frac{x-x_{\nu}}{h}\right)\left[f^{(2 r+1)}\left(x_{\nu}\right)-f^{(2 r+1)}\left(x_{0}\right)\right] h^{2 r+1-q} \\
& +O\left(h^{2 r+1-q} \omega\left(f^{(2 r+2)} ; h\right)\right) .
\end{aligned}
$$

最后, 设 $2(r-k) \leqslant s \leqslant 2 r$. 为了叙述简单, 令 $s$ 为一偶数. 此时

$$
\begin{aligned}
& h^{2 i+1} S_{n}^{(2 i+1)}\left(x_{\nu+1}-\right)=\sum_{p=0}^{\frac{s}{2}} h^{2 p}\left[f^{(2 p)}\left(x_{v}\right) v_{0, p}^{(2 i+1)}(1)+f^{(2 p)}\left(x_{\nu+1}\right) v_{1, p}^{(2 i+1)}(1)\right] \\
& +\sum_{p=r-k+1}^{\frac{s}{2}} h^{2 p}\left\{\left[S_{n}^{(2 p)}\left(x_{\nu}+\right)-f^{(2 p)}\left(x_{\nu}\right)\right] v_{0, p}^{(2 i+1)}(1)\right. \\
& \left.+\left[S_{n}^{(2 p)}\left(x_{\nu+1}-\right)-f^{(2 p)}\left(x_{\nu+1}\right)\right] v_{1, p}^{(i)+1)}(1)\right\} \\
& +\sum_{p=\frac{s}{2}+1}^{r} h^{2 p}\left[S_{n}^{(1 p)}\left(x_{\nu}+\right) v_{0, p}^{(2 i+1)}(1)+S_{n}^{(1 p)}\left(x_{\nu+1}-\right) v_{1, p}^{(2 i+1)}(1)\right] .
\end{aligned}
$$

对于 $h^{2 i+1} S_{n}^{(2 i+1)}\left(x_{v+1}+\right)$ 也有类似关系式, 故有连续性方程

$$
\begin{aligned}
& \sum_{p=r-k+1}^{s} h^{2 p}\left\{\left[S_{n}^{(2 p)}\left(x_{\nu}+\right)-f^{(2 p)}\left(x_{\nu}\right)+S_{n}^{(2 p)}\left(x_{\nu+2}-\right)-f^{(2 p)}\left(x_{\nu+2}\right)\right] v_{0, p}^{(2 i+1)}(1)\right. \\
& \left.\quad+\left[S_{n}^{(2 p)}\left(x_{\nu+1}-\right)-f^{(2 p)}\left(x_{\nu+1}\right)+S_{n}^{(2 p)}\left(x_{\nu+1}+\right)-f^{(2 p)}\left(x_{\nu+1}\right)\right] v_{1, p}^{(2 i+1)}(1)\right\} \\
& \quad+\sum_{p=\frac{s}{2}+1}^{s} h^{2 p}\left\{\left[S_{n}^{(2 p)}\left(x_{\nu}+\right)-S_{n}^{(2 p)}\left(x_{\nu+2}-\right)\right] v_{0, p}^{(2 i+1)}(1)\right. \\
& \left.\quad+\left[S_{n}^{(2 p)}\left(x_{\nu+1}-\right)-S_{n}^{(2 p)}\left(x_{\nu+1}+\right)\right] v_{1, p}^{(2 i+1)}(1)\right\} \\
& =\sum_{p=0}^{s / 2} h^{2 p}\left\{\left[f^{(2 p)}\left(x_{\nu+1}\right) v_{0, p}^{(2 i+1)}(0)+f^{(2 p)}\left(x_{\nu+2}\right) v_{1, p}^{(2 i+1)}(0)\right]\right. \\
& \left.\quad-\left[f^{(2 p)}\left(x_{\nu}\right) v_{0, p}^{(2 i+1)}(1)+f^{(2 p)}\left(x_{\nu+1}\right) v_{1, p}^{(2 i+1)}(1)\right]\right\} . \quad(\nu=1,2, \cdots, n-1)
\end{aligned}
$$

把

$$
f^{(2 p)}\left(x_{v+2}\right)=\sum_{k=2 p}^{s-1} \frac{f^{(k)}\left(x_{v+1}\right)}{(k-2 p) !} h^{k-2 p}+\frac{f^{(s)}\left(\xi_{p, v+1}\right)}{(s-2 p) !} h^{s-2 p}
$$

代入(12)式右端, 并利用(4)式, 不难得到 


$$
\begin{aligned}
& \sum_{p=0}^{s / 2} h^{2 p}\left[f^{(2 p)}\left(x_{\nu+1}\right) v_{0, p}^{(2 i+1)}(0)+f^{(2 p)}\left(x_{\nu+2}\right) v_{1, p}^{(2 i+1)}(0)\right] \\
= & h^{2 i+1} f^{(2 i+1)}\left(x_{\nu+1}\right)+O\left(h^{s} \omega\left(f^{(s)} ; h\right)\right) .
\end{aligned}
$$

同理

$$
\begin{aligned}
& \sum_{p=0}^{s / 2} h^{2 p}\left[f^{(2 p)}\left(x_{v}\right) v_{0, p}^{(2 i+1)}(1)+f^{(2 p)}\left(x_{v+1}\right) v_{1, p}^{(2 i+1)}(1)\right] \\
= & h^{2 i+1} f^{(2 i+1)}\left(x_{v+1}\right)+O\left(h^{s} \omega\left(f^{(s)} ; h\right)\right) .
\end{aligned}
$$

即(12)式右端等于 $O\left(h^{s} \omega\left(f^{(s)} ; h\right)\right)$. 用前面类似推导过程可得

$$
\left\{\begin{array}{l}
S_{n}^{(2 p)}\left(x_{\nu} \pm\right)-f^{(2 p)}\left(x_{\nu}\right)=O\left(h^{s-2 p-1} \omega\left(f^{(s)} ; h\right)\right), p \leqslant \frac{s}{2}, \\
S_{n}^{(2 p)}\left(x_{\nu} \pm\right)=O\left(h^{s-2 p-1} \omega\left(f^{(s)} ; h\right)\right) . \quad p>\frac{s}{2} .
\end{array}\right.
$$

由于当 $x \in\left(x_{v}, x_{v+1}\right)$ 时, $S_{n}^{(s)}(x)$ 为次数不高于 $2 r+1-s$ 次多项式, 故

$$
S_{n}^{(s)}(x)=\sum_{p=0}^{-\frac{s}{2}}\left[S_{n}^{(s+2 p)}\left(x_{v}+\right) v_{0, p}^{*}\left(\frac{x-x_{p}}{h}\right)+S_{n}^{(s+2 p)}\left(x_{v+1}-\right) v_{1, p}^{*}\left(\frac{x-x_{p}}{h}\right)\right] h^{2 p},
$$

其中 $v_{i, p}^{*}(x)$ 为对应的 $2\left(r-\frac{s}{2}\right)+1$ 次插值基函数. 于是

$$
\begin{aligned}
S_{n}^{(s)}(x) & -f^{(s)}(x)=\left[S_{n}^{(s)}\left(x_{v}+\right)-f^{(s)}\left(x_{v}\right)+f^{(s)}\left(x_{v}\right)-f^{(s)}(x)\right] v_{0,0}^{*}\left(\frac{x-x_{v}}{h}\right) \\
& +\left[S_{n}^{(s)}\left(x_{v+1}-\right)-f^{(s)}\left(x_{v+1}\right)+f^{(s)}\left(x_{v+1}\right)-f^{(s)}(x)\right] v_{1,0}^{*}\left(\frac{x-x_{v}}{h}\right) \\
& +\sum_{p=1}^{r-\frac{s}{2}} h^{2 p}\left[S_{n}^{(s+2 p)}\left(x_{v}+\right) v_{0, p}^{*}\left(\frac{x-x_{v}}{h}\right)\right. \\
& \left.+S_{n}^{(s+2 p)}\left(x_{v+1}-\right) v_{1, p}^{*}\left(\frac{x-x_{v}}{h}\right)\right] .
\end{aligned}
$$

把(13)式代人即得 $S_{n}^{(s)}(x)-f^{(s)}(x)=O\left(h^{s-1} \omega\left(f^{(s)} ; h\right)\right)$. 应用 Rolle 定理即可证得定理.

系 设 $\left\|f^{(2 r+2)}(x)\right\| \leqslant M$, 则 $\left\|S_{n}^{(q)}(f ; x)-f^{(q)}(x)\right\|=O\left(n^{-(2 r+1)+q}\right)$. 如

$$
f(x) \in C^{2 r+2}[0,1] \text {, }
$$

则当 $\left\|S_{n}^{(q)}(f ; x)-f^{(q)}(x)\right\|=o\left(n^{-(2 r+1)+q}\right)$ 对某个 $q$ 成立时, $f(x)$ 是一个次数不高于 $2 r+1$ 的多项式.

这样, 我们在很一般的条件下 (许多具体的样条都满足), 完全解决了这类缺插值样条函数 的收玫速度估计、渐近展开、饱和度和饱和函数类等问题, 类似的其它类型(如 $r=3 k-1$ ) 以 及其它端点条件均可用同样方法讨论,这里不再刋述.

\section{参考文 嶰}

[1] Meir, A. \& Sharma, A., SIAM. J. Numer. Anal., 10 (1973), 433-442.

[2] Swař, B. K. \& Varga, B. S., ibid, 10 (1973), 443-447.

[3] 部竹瑞, 数学学报, 18 (1975), 247-253.

[4] 陈天平,中国科学, 1980, 12: 1137-1146. 